\title{
A RESULT ON MONOTONICALLY LINDELÖF GENERALIZED ORDERED SPACES
}

\author{
AI-JUN XU and WEI-XUE SHI ${ }^{凶}$
}

(Received 26 March 2011)

\begin{abstract}
In this paper, we show that the character of any monotonically Lindelöf generalized ordered (GO) space is not greater than $\omega_{1}$, which gives a negative answer to a question posed by Levy and Matveev ['Some questions on monotone Lindelöfness', Questions Answers Gen. Topology 26 (2008), 13-27, Question 51].

2010 Mathematics subject classification: primary 54F05; secondary 54D20.

Keywords and phrases: monotonically Lindelöf, generalized ordered spaces.
\end{abstract}

\section{Introduction}

A topological space $X$ is monotonically Lindelöf if there is an operator $r$ which assigns to every open cover $\mathcal{U}$ of $X$ a countable open cover $r \mathcal{U}$ of $X$ that refines $\mathcal{U}$ such that if $\mathcal{U}$ refines $\mathcal{V}$ then $r \mathcal{U}$ refines $r \mathcal{V}$. In [1, Example 2.2, Corollary 2.4], Bennett et al. proved that a monotonically Lindelöf linearly ordered topological space (LOTS) need not be first countable and any monotonically Lindelöf compact LOTS is first countable. So, Levy and Matveev posed the following question.

Question [4, Question 51]. Can the character of a monotonically Lindelöf generalized ordered space be greater than $\omega_{1}$ ?

In this paper, we give a negative answer to this question.

For undefined terms and notation we refer to $[2,5,7]$.

\section{Main results}

Definition 2.1 [6]. Let $L$ be a compact LOTS. For $x \in L$, put

$$
0-\operatorname{cf}(x)=\min \{|C|: C \text { is a cofinal subset of }(\leftarrow, x)\}
$$

and

$$
1-\operatorname{cf}(x)=\min \{|C|: C \text { is a coinitial subset of }(x, \rightarrow)\} ;
$$

$0-\operatorname{cf}(x)$ denotes the left cofinality of $x$, and 1-cf $(x)$ denotes the right one of $x$.

This work is supported by NSFC (no:10971092), NJFU (no:163101088).

(C) 2011 Australian Mathematical Publishing Association Inc. 0004-9727/2011 \$16.00 
Note that $0-\operatorname{cf}(x)$ and $1-\operatorname{cf}(x)$ are dual notions, so we only discuss the left cofinality $0-\operatorname{cf}(x)$ in the following. For a compact LOTS $L$, observe that $0-\operatorname{cf}(x)=0$ if $x$ is the left endpoint of $L, 0-\operatorname{cf}(x)=1$ if $x$ has an immediate predecessor in $L$, and $0-\operatorname{cf}(x)$ is a regular cardinal otherwise. It is well known that a GO-space $X$ can be embedded as a dense subspace into the compact LOTS $l(X)$ that is called the minimal linearly ordered compactification of $X$ (see [3]). For a GO-space $X$ and $x \in X$, the left cofinality $0-\operatorname{cf}(x)$ means the cofinality defined in its minimal linearly ordered compactification $l(X)$. By [3, Lemma 3.5], if $0-\operatorname{cf}(x) \geq \omega$, then there exists a cofinal increasing sequence $\left\{x_{0}(\alpha) \in X \mid \alpha<0-\operatorname{cf}(x)\right\}$. In addition, $x$ has an immediate predecessor or $[x, \rightarrow)$ is open in $X$ if $0-\operatorname{cf}(x)=1$.

The next definition was introduced by Matveev.

Definition 2.2. Let $x$ be a point of a space $X$. Then $X$ is said to be monotonically Lindelöf at $x$ if there exists an operator $r_{x}$ that assigns to every nonempty family $\mathcal{F}$ of neighborhoods of $x$ a nonempty countable family $r_{x} \mathcal{F}$ of neighborhoods of $x$ so that $r_{x} \mathcal{F}$ refines $\mathcal{F}$ and $r_{x} \mathcal{F}$ refines $r_{x} \mathcal{G}$ provided that $\mathcal{F}$ refines $\mathcal{G}$. In this case, $r_{x}$ is called a monotone Lindelöf operator at the point $x$ of $X$.

In [1, Example 2.3], Bennett et al. proved that [0, $\left.\omega_{1}\right]$ considered as a LOTS is not monotonically Lindelöf. With a slight modification of the proof of [1, Example 2.3], we have the following lemma.

Lemma 2.3. Suppose that $\kappa>\omega_{1}$ is a regular ordinal and $S$ is a stationary subset of $[0, \kappa)$. Then $S \cup\{\kappa\}$ considered as a LOTS is not monotonically Lindelöf.

Theorem 2.4. Suppose that X is a GO-space. If X is monotonically Lindelöf, then both the left and right cofinalities at each point $x$ of $X$ are not greater than $\omega_{1}$.

Proof. We only prove the 'left' case; the other case can be proved similarly.

Suppose instead that the left cofinality $0-\operatorname{cf}(x)$ of $X$ at $x$ is greater than $\omega_{1}$. Take a cofinal increasing sequence $S_{0}(x)=\left\{x_{0}(\alpha) \in X \mid \alpha<0-\operatorname{cf}(x)\right\}$. Without loss of generality, we may assume that $S_{0}(x)$ is closed in $(\leftarrow, x)$. Then $S_{0}(x)$ must be homeomorphic to a subspace $H$ of $[0,0-\operatorname{cf}(x))$. If $H$ is a stationary subset, then $H \cup\{0-\operatorname{cf}(x)\}$ is not monotonically Lindelöf by Lemma 2.3. Hence, $S_{0}(x) \cup\{x\}$ is not monotonically Lindelöf. However, this contradicts that $S_{0}(x) \cup\{x\}$ is a closed subspace of the monotonically Lindelöf space $X$. Therefore $H$ is not stationary in $[0,0-\operatorname{cf}(x))$. It follows that there exists a closed cofinal subset $C$ of $[0,0-\operatorname{cf}(x))$ such that $C \cap H=\emptyset$. Thus we conclude that $H$ can be presented as a union of $0-\operatorname{cf}(x)$ many pairwise disjoint open convex subsets of $H$ and so can $S_{0}(x)$ in $(\leftarrow, x)$. Hence we may write

$$
S_{0}(x)=\bigcup\left\{T_{\alpha} \mid \alpha<0-\operatorname{cf}(x)\right\}
$$

where $T_{\alpha}$ is convex and open, and if $x^{\prime} \in T_{\alpha}, x^{\prime \prime} \in T_{\gamma}$ for $\alpha<\gamma$, then $x^{\prime}<x^{\prime \prime}$. Since $0-\operatorname{cf}(x)>\omega_{1}$, the set $\mathcal{U}=\left\{T_{\alpha} \mid \alpha<\omega_{1}\right\} \cup\left\{y \in S_{0}(x) \cup\{x\} \mid y \geq a\right.$ for every $\left.a \in T_{\omega_{1}}\right\}$ is an open cover of $S_{0}(x) \cup\{x\}$ that has no countable subcover. Thus $S_{0}(x) \cup\{x\}$ is not Lindelöf. We obtain a contradiction. 
THEOREM 2.5. The character of any monotonically Lindelöf $G O$-space $(X, \tau)$ is not greater than $\omega_{1}$.

Proof. Suppose that $(X, \tau)$ is a monotonically Lindelöf GO-space. Then, for every $x \in X$, neither the left nor the right cofinalities of $x$ is greater than $\omega_{1}$ by Theorem 2.4. We only consider the case that both the left and the right cofinalities of $x$ are $\omega_{1}$. The other cases are easy. Let $\left\{a_{\gamma} \mid \gamma<\omega_{1}\right\}$ be a cofinal increasing sequence of $(\leftarrow, x)$ and $\left\{b_{\gamma} \mid \gamma<\omega_{1}\right\}$ a coinitial decreasing sequence of $(x, \rightarrow)$. Put

$$
\mathcal{B}(x)=\left\{\left(a_{\gamma}, b_{\gamma}\right) \mid \gamma<\omega_{1}\right\} .
$$

Then $\mathcal{B}(x)$ is a base for $(X, \tau)$ at the point $x$ and $|\mathcal{B}(x)| \leq \omega_{1}$. Hence $\chi(x,(X, \tau)) \leq \omega_{1}$. In view of the arbitrariness of $x$, the character of GO-space $(X, \tau)$ is not greater than $\omega_{1}$.

\section{Acknowledgement}

The authors would like to express their thanks to the referee for valuable suggestions and comments that improved this paper.

\section{References}

[1] H. Bennett, D. Lutzer and M. Matveev, 'The monotone Lindelöf property and separability in ordered spaces', Topology Appl. 151 (2005), 180-186.

[2] R. Engleking, General Topology, revised and completed edition, Sigma Series in Pure Mathematics, 6 (Hedermann, Berlin, 1989).

[3] N. Kemoto, 'Normality of products of GO-spaces and cardinals', Topology Proc. 18 (1993), 133-142.

[4] R. Levy and M. Matveev, 'Some questions on monotone Lindelöfness', Questions Answers Gen. Topology 26 (2008), 13-27.

[5] D. Lutzer, 'Ordered topological spaces', in: Surveys in General Topology (ed. G. M. Reed) (Academic Press, New York, 1980), pp. 247-296.

[6] A.-J. Xu and W.-X. Shi, 'Notes on monotone Lindelöf property', Czechoslovak Math. J. 59(134) (2009), 943-955.

[7] A.-J. Xu and W.-X. Shi, 'Monotone Lindelöf property and linearly ordered extensions', Bull. Aust. Math. Soc. 81 (2010), 418-424.

\section{AI-JUN XU, School of Mathematical Sciences, Nanjing Normal University, Nanjing 210046, PR China \\ e-mail: ajxu@njfu.edu.cn}

WEI-XUE SHI, Department of Mathematics, Nanjing University, Nanjing 210093, PR China

e-mail:wxshi@nju.edu.cn 